\title{
Avaliação do Código Computacional DEPP na Otimização de Problemas de Propulsão e Aerodinâmica de Foguetes
}

\author{
Jonas J. Radtke, Guilherme Bertoldo, \\ Universidade Tecnológica Federal do Paraná \\ 85601-970, Francisco Beltrão, PR \\ E-mail: jonas@utfpr.edu.br e gbertoldo@utfpr.edu.br \\ Carlos H. Marchi \\ Universidade Federal do Paraná - Departamento de Engenharia Mecânica \\ 81531-980, Curitiba, PR \\ E-mail:marchi@ufpr.br
}

\begin{abstract}
Resumo: No presente trabalho foi implementado um código computacional para a otimização global de um conjunto arbitrário de variáveis. $O$ código computacional, denominado DEPP (Differential Evolution Parallel Program), é baseado no algoritmo de Evolução Diferencial e utiliza o Método de Superfície de Resposta para aceleração da convergência. O DEPP foi implementado em linguagem Fortran 95 e utiliza as diretivas do MPI (Message Passing Interface) para processamento paralelo. A aptidão de cada indivíduo é obtida por um programa auxiliar. A verificação do DEPP foi realizada utilizando as funções de Ackley e Rastrigin, com até cinco variáveis. Problemas aplicados à propulsão e aerodinâmica de foguetes foram considerados. As geometrias de uma tubeira e do nariz de um foguete foram otimizadas. A avaliação da aptidão de cada geometria da tubeira gerada pelo DEPP foi realizada pelo código Mach2D, que simula o escoamento no interior da tubeira, utilizando o método de volumes finitos. O DEPP convergiu para as soluções exatas tanto para as funções de Ackley e Rastrigin, como na otimização aerodinâmica de Newton. O desempenho da Evolução Diferencial com hibridização foi superior ao algoritmo puro. Na otimização da geometria de tubeiras, o DEPP determinou uma geometria que fornece coeficiente de empuxo superior ao obtido pelo Método das Características.
\end{abstract}

Palavras-chave: Otimização. Evolução Diferencial. Geometria. Propulsão. Aerodinâmica. Foguete.

\section{Introdução}

Problemas que envolvem otimização global sobre espaços contínuos são muito comuns na comunidade científica. O primeiro passo na otimização de um problema consiste em escolher uma função que modela os objetivos do problema. Sobre as variáveis desta função podem ser aplicadas quaisquer restrições. Quando a função objetivo é não linear e não diferenciável, as abordagens com busca direta são mais indicadas [6].

O algoritmo de Evolução Diferencial (DE) é uma estratégia de busca direta bastante conhecida, apesar de ter sido desenvolvida muito recentemente [3]. Dentre as vantagens da DE, destaca-se a robustez, boas propriedades de convergência e a habilidade em lidar com funções objetivos não diferenciáveis e não lineares. A grande facilidade de uso e de paralelização são outras características importantes desta metodologia.

Em diversos problemas de otimização não é possível determinar uma expressão para a função objetivo. A otimização da geometria em problemas de propulsão e aerodinâmica de foguetes são exemplos disto. Neste trabalho o coeficiente de empuxo e o coeficiente de arrasto foram utilizados para mensurar a qualidade da geometria, para os problemas de propulsão e aerodinâmica, respectivamente. 


\section{Método de Otimização}

DE é um algoritmo evolucionário, isto é, ele realiza a evolução de uma população de indivíduos de alguma maneira inteligente. A geração de novos indivíduos é obtida pelo uso de diferenças entre indivíduos da população atual, utilizando uma forma simples e rápida chamada operador diferencial [3].

O algoritmo DE é inicializado com um conjunto de palpites para a solução do problema. Cada palpite, chamado indivíduo, é dado por um vetor composto por valores para todas as variáveis da função objetivo, denominados cromossomos. O conjunto de todos os indivíduos é chamado de população.

A população inicial é obtida aleatoriamente. Cada nova população é gerada com base nos cromossomos e na aptidão dos indivíduos da população anterior, utilizando operadores diferenciais. Cada cromossomo de um novo indivíduo pode, ou não, ser modificado (mutação) com uma dada probabilidade. $\mathrm{O}$ valor de cada cromossomo é dado por uma combinação linear dos valores dos cromossomos de dois indivíduos escolhidos aleatoriamente.

Para fazer todas as simulações numéricas foram utilizadas populações com tamanho igual a dez vezes o número de incógnitas. A constante de diferenciação e a probabilidade de mutação foram tomadas iguais a 0,85 e 0,5 , respectivamente. O Método de Superfície de Resposta (RSM) foi utilizado para aceleração da convergência.

A hibridização do algoritmo DE com o RSM consiste em determinar os valores dos cromossomos de forma que o indivíduo gerado seja um ótimo, dentre um conjunto de indivíduos com as melhores aptidões. Para tanto, uma função quadrática é ajustada aos melhores resultados da população atual. O ponto de máximo é obtido desta função e as coordenadas deste ponto são utilizadas como cromossomos de um indivíduo da próxima geração [7].

O código computacional desenvolvido no presente trabalho, denominado DEPP, é apto a resolver qualquer problema de otimização global. Um programa serial auxiliar é responsável pelo cálculo da aptidão de cada indivíduo gerado pelo DEPP, o que torna fácil a aplicação deste programa a qualquer problema, apenas criando um programa auxiliar específico para o problema que se deseja resolver.

O DEPP foi implementado em linguagem Fortran 95 e utiliza as diretivas do MPI (Message Passing Interface) para processamento paralelo. O código-fonte do DEPP está disponível no seguinte endereço: http://depp.googlecode.com/svn/trunk. Neste mesmo endereço está o código-fonte do programa auxiliar, utilizado para calcular a aptidão das funções teste. A revisão 39 do DEPP foi utilizada para a obtenção dos resultados apresentados neste trabalho.

\section{Verificação}

Para verificação do DEPP foram utilizadas as funções testes de Ackley e Rastrigin. Tais funções são problemas clássicos destinados a avaliar a eficiência e convergência de algoritmos de busca. Ambas as funções testes possuem vários ótimos locais, o que dificulta a obtenção do ótimo global por métodos baseados em gradientes. As expressões das funções de Ackley e Rastrigin são dadas, respectivamente, por [3]:

$$
f=20 \exp \left(-0,2 \sqrt{\frac{1}{n} \sum_{i=1}^{n} x_{i}^{2}}\right)+\exp \left(\frac{1}{n} \sum_{i=1}^{n} \cos \left(2 \pi x_{i}\right)\right)-20-\exp (1),-32,7 \leq x_{i} \leq 32,7
$$

$\mathrm{e}$

$$
f=-\sum_{i=1}^{n}\left(x_{i}^{2}-10 \cos \left(2 \pi x_{i}\right)+10\right), \quad-5,12 \leq x_{i} \leq 5,12
$$


A otimização das funções de Ackley e Rastrigin foram simuladas com até cinco incógnitas. As Figuras 1 e 2 mostram que a solução obtida pelo DEPP converge para o ótimo global em todos os casos. Em todos os casos avaliados o desempenho do DEPP com a aplicação da hibridização com o RSM é melhor do que sem o uso desta técnica.

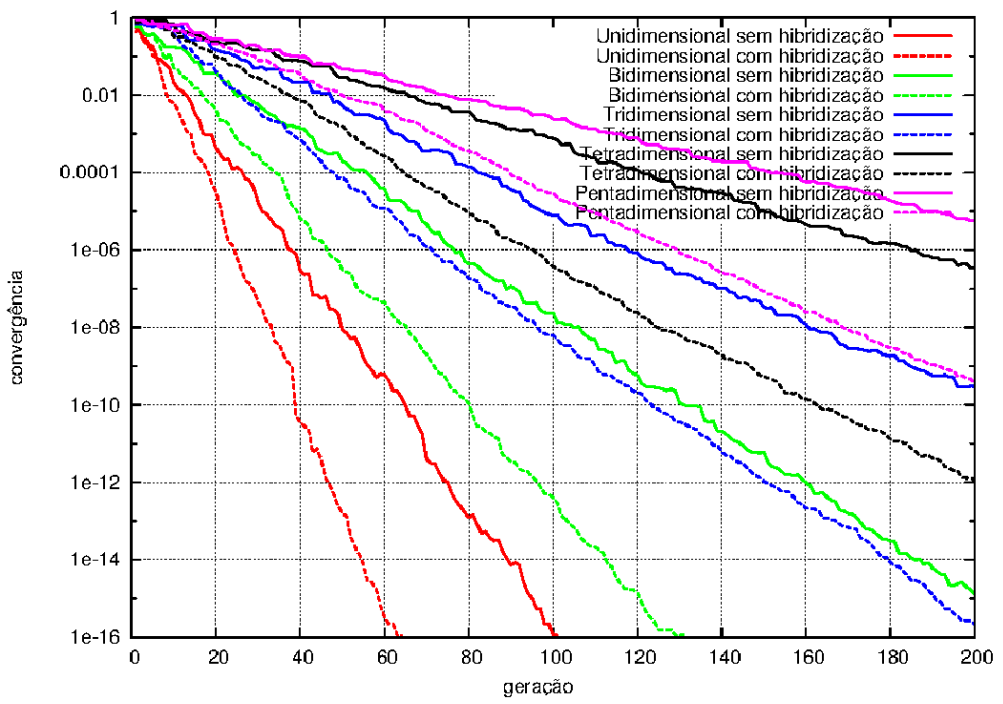

Figura 1: Desempenho do DEPP na otimização da função de Ackley.

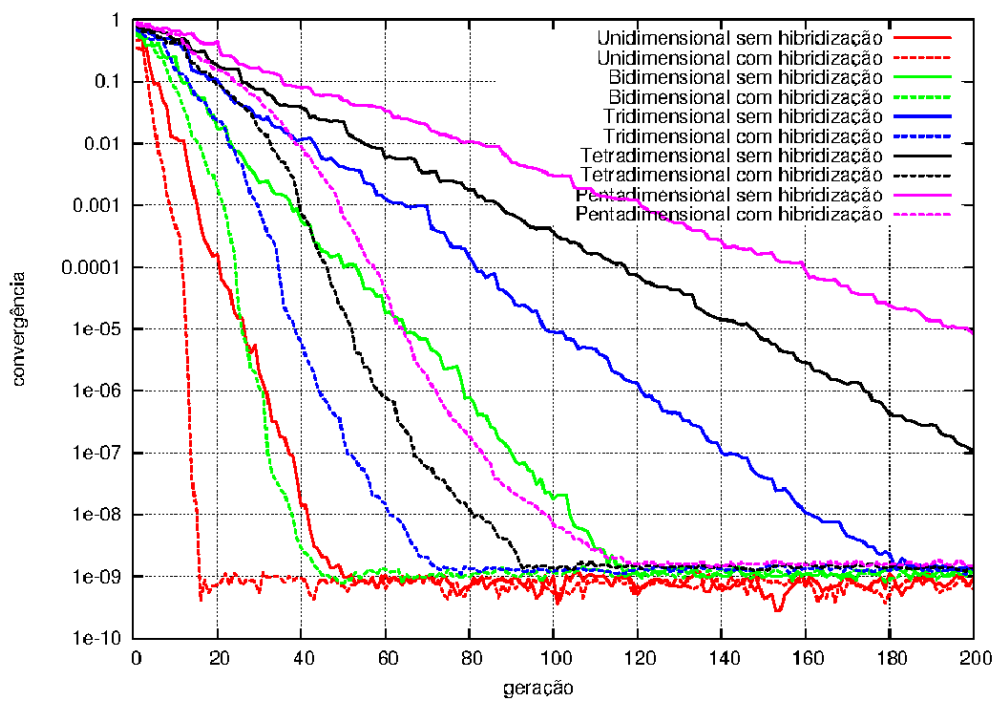

Figura 2: Desempenho do DEPP na otimização da função de Rastrigin.

\section{Otimização Aerodinâmica de Newton}

O problema de otimização de Newton [5], para um escoamento axial, consiste em determinar o perfil geométrico $y(x)$ de um corpo com simetria axial com comprimento unitário e diâmetro $1 / f$ prescrito que minimiza o coeficiente de arrasto frontal $C_{D f}$. Newton resolveu o problema considerando que a distribuição de pressão sobre a geometria depende apenas da inclinação local da superfície (fórmula de pressão de Newton [1]) e mostrou que a forma otimizada deve possuir uma face plana seguida por um arco suave (Figura 3). 


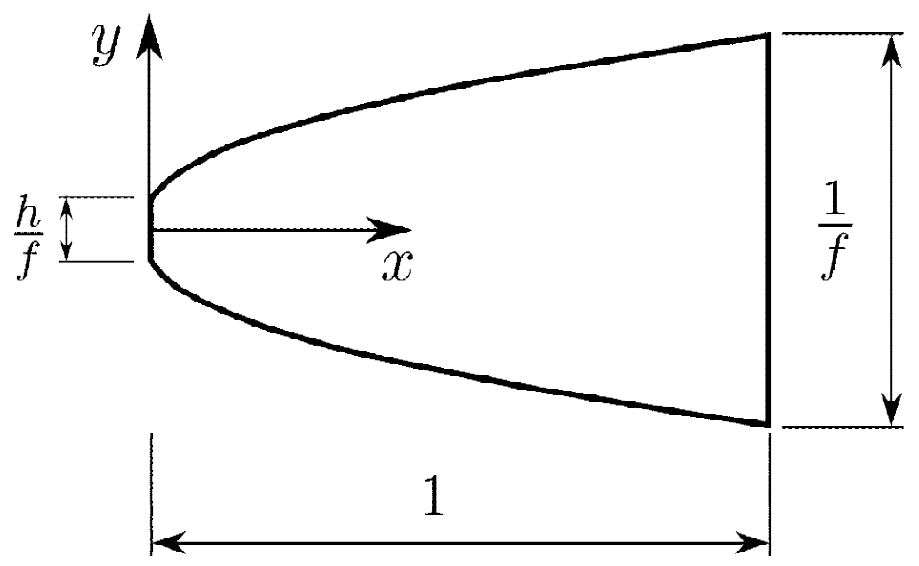

Figura 3: Esboço do perfil geométrico otimizado de Newton.

A forma otimizada de Newton é dada parametricamente por

$$
x(\zeta)=\frac{p(\zeta)-p\left(\zeta_{f}\right)}{p\left(\zeta_{b}\right)-p\left(\zeta_{f}\right)}, \quad \zeta_{b} \leq \zeta \leq \zeta_{f}
$$

e

$$
y(\zeta)=\frac{(h-1) w(\zeta)+w\left(\zeta_{f}\right)-h w\left(\zeta_{b}\right)}{2 f\left[w\left(\zeta_{f}\right)-w\left(\zeta_{b}\right)\right]}, \quad \zeta_{b} \leq \zeta \leq \zeta_{f}
$$

onde

$$
\begin{gathered}
\zeta=\frac{d y}{d x} \\
p(\zeta)=\frac{3}{4 \zeta^{4}}+\frac{1}{\zeta^{2}}+\ln \zeta
\end{gathered}
$$

e

$$
w(\zeta)=\frac{\left(1+\zeta^{2}\right)^{2}}{\zeta^{3}}
$$

Nas Eqs. (3) e (4), $h$ representa uma fração do diâmetro da base, $\zeta_{f}$ a inclinação do perfil geométrico na parte frontal e $\zeta_{b}$ a inclinação na base. Para a forma otimizada de Newton, $\zeta_{f}=1$, enquanto $h$ e $\zeta_{b}$ são determinados a partir das seguintes relações:

$$
(h-1)\left[p\left(\zeta_{b}\right)-p\left(\zeta_{f}\right)\right]=2 f\left[w\left(\zeta_{f}\right)-w\left(\zeta_{b}\right)\right]
$$

e

$$
h=\frac{w\left(\zeta_{f}\right)}{w\left(\zeta_{b}\right)} .
$$


O objetivo deste teste é avaliar a eficiência do código DEPP ao ser aplicado em um problema de otimização aerodinâmica. Para isso, os parâmetros $\zeta_{f}$ e $h$ foram considerados variáveis de otimização e determinados de modo a minimizar o coeficiente de arrasto frontal, dado por:

$$
C_{D f}=16 f^{2} \int_{\zeta_{b}}^{\zeta_{f}} \frac{\zeta^{2}}{1+\zeta^{2}} y(\zeta) \frac{d y}{d \zeta} d \zeta+2 h^{2}
$$

Espera-se que a solução da otimização se aproxime dos valores exatos de $\zeta_{f}$ e $h$.

As otimizações foram realizadas considerando-se os seguintes intervalos para as variáveis $\zeta_{f}$ e $h$ :

$$
0,5 \leq \zeta_{f} \leq 1,6 \text { e } 10^{-4} \leq h \leq 0,2 .
$$

As gerações foram interrompidas quando $\varepsilon \leq 10^{-5}$, sendo $\varepsilon$ uma medida de convergência da população (P-measure) definida na Ref. [3]. Três razões de aspectos foram analisadas: $f=2,3$ e 4 . As otimizações foram realizadas em triplicata para cada razão de aspecto. A solução final é dada pela média das soluções em cada otimização.

A Tabela 1 apresenta as soluções exatas e as soluções aproximadas obtidas com os algoritmos DE e DE-RSM, bem como, o número médio de gerações $(g)$ necessárias para atingir a tolerância prescrita. Como se pode observar, tanto o algoritmo puro (DE), quanto o hibridizado (DE-RSM) convergiram para a solução exata dentro da tolerância prescrita. Além disso, o número de gerações do algoritmo hibridizado foi cerca de $45 \%$ menor que o algoritmo puro.

Tabela 1: Comparação entre as variáveis otimizadas com o DEPP e a solução exata da otimização aerodinâmica de Newton.

\begin{tabular}{|c|l|c|c|c|c|}
\hline$f$ & Método & $\zeta_{f}$ & $h$ & $C_{D f}$ & $g$ \\
\hline \multirow{3}{*}{2} & Exato & 0,02277468748040 & 1,00000000000000 & 0,09815945704287 & \\
\cline { 2 - 6 } & DE & 0,02277470623297 & 0,99999971583410 & 0,09815945704287 & 91 \\
\cline { 2 - 6 } & DE-RSM & 0,02277468580303 & 0,99999981420069 & 0,09815945704287 & 51 \\
\hline \multirow{3}{*}{3} & Exato & 0,00732592998492 & 1,00000000000000 & 0,04540987044228 & \\
\cline { 2 - 7 } & DE & 0,00732593240297 & 1,00000073866375 & 0,04540987044228 & 95 \\
\cline { 2 - 7 } & DE-RSM & 0,00732592920496 & 1,00000132991582 & 0,04540987044228 & 55 \\
\hline \multirow{3}{*}{4} & Exato & 0,00317982554119 & 1,00000000000000 & 0,02590256872307 & \\
\cline { 2 - 6 } & DE & 0,00317982548704 & 1,00000164785640 & 0,02590256872307 & 131 \\
\cline { 2 - 6 } & DE-RSM & 0,00317982248807 & 1,00000128141432 & 0,02590256872307 & 67 \\
\hline
\end{tabular}

\section{Otimização da Geometria de Tubeiras}

No problema aplicado à propulsão de foguetes foi utilizado um código computacional chamado Mach2D para simular o escoamento em cada geometria gerada pelo DEPP. O Mach2D simula o escoamento no interior da tubeira e determina o coeficiente de empuxo, que é tomado como a aptidão da geometria.

Para simular o escoamento foi utilizado um modelo bidimensional axissimétrico. O modelo é baseado nas equações da conservação da massa, da quantidade de movimento linear (equação de Euler), da energia, e na equação de estado, dadas respectivamente por: 


$$
\begin{gathered}
\frac{\partial(\rho u)}{\partial x}+\frac{1}{y} \frac{\partial(y \rho v)}{\partial y}=0 \\
\frac{\partial\left(\rho u^{2}\right)}{\partial x}+\frac{1}{y} \frac{\partial(y \rho u v)}{\partial y}+\frac{\partial p}{\partial x}=0 \\
\frac{\partial(\rho u v)}{\partial x}+\frac{1}{y} \frac{\partial\left(y \rho v^{2}\right)}{\partial y}+\frac{\partial p}{\partial y}=0 \\
c_{p} \frac{\partial(\rho T u)}{\partial x}+\frac{c_{p}}{y} \frac{\partial(y \rho T v)}{\partial y}+\frac{\partial(p u)}{\partial x}+\frac{1}{y} \frac{\partial(y p v)}{\partial y}=0 \\
p=\rho R T
\end{gathered}
$$

sendo $\rho, u, v, p$ e $T$ as variáveis dependentes, representando a massa específica, a velocidade axial, a velocidade radial, a pressão e a temperatura, respectivamente; $x$ e $y$, nesta ordem, as direções axial e radial.

Sob o sistema de equações de conservação são aplicadas condições de contorno a fim de solucionar de forma única o problema. As condições de contorno utilizadas neste trabalho são apresentadas na Figura 4.

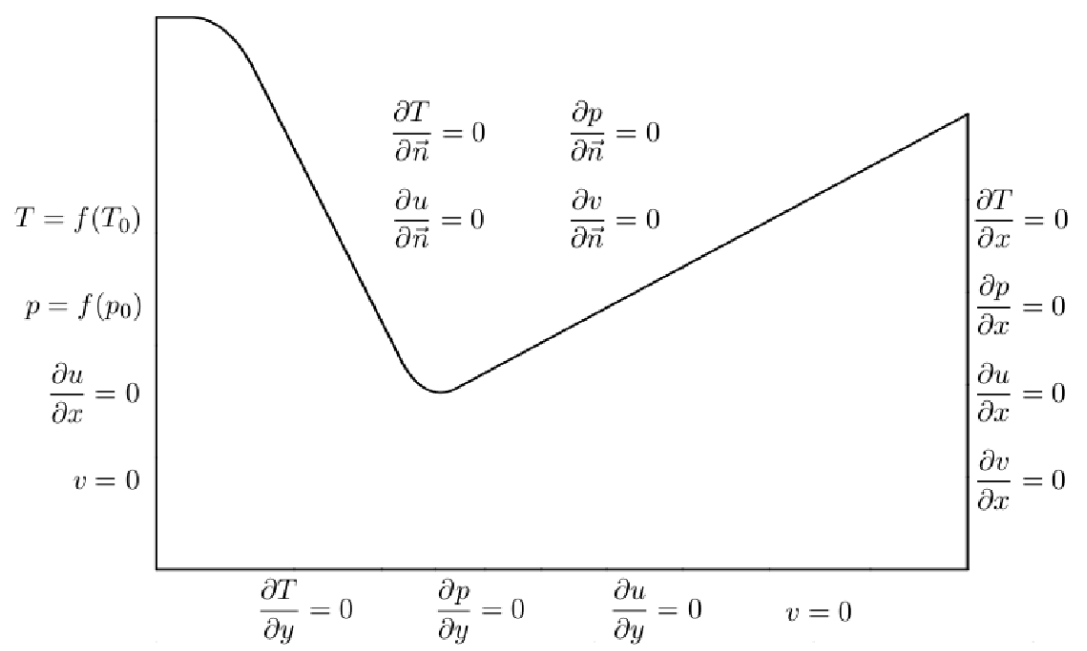

Figura 4: Condições de contorno aplicadas ao escoamento no interior da tubeira.

O modelo numérico utilizado neste trabalho é baseado no Método de Volumes Finitos com arranjo co-localizado de variáveis [4]. Volumes fictícios são utilizados para a aplicação das condições de contorno. Funções de interpolação de primeira e segunda ordem (UDS e CDS) são utilizadas para calcular as derivadas do modelo matemático. O escoamento foi simulado utilizando malhas com 480 volumes na direção axial e 240 volumes na direção radial.

A geometria da seção divergente da tubeira foi construída utilizando-se um Spline contendo entre 1 e 4 pontos de controle. A inclinação da parede na saída também foi tomada como variável na otimização. O comprimento da seção divergente da tubeira foi tomado igual a 1 metro e os raios da garganta, da curvatura na garganta e da saída iguais a 5, 1 e 27,1743 centímetros, respectivamente.

Nesta etapa foram utilizadas 50 gerações no DEPP. Os resultados obtidos foram comparados com o coeficiente de empuxo de uma tubeira obtida pelo Método das 
Características $\left(C_{F v}^{*}\right)$, com mesmo comprimento, raio da garganta e razão de expansão [2]. Os resultados obtidos pelo DEPP são apresentados na Tabela 2.

Tabela 2: Comparação entre os coeficiente de empuxo otimizado pelo DEPP e o fornecido pelo Método das Características.

\begin{tabular}{|c|c|c|}
\hline Variáveis & Coef. de empuxo $\left(C_{F v}\right)$ & $C_{F v} / C_{C v}^{*}$ \\
\hline 2 & 1,8735320920 & 0,999940262 \\
\hline 3 & 1,8758986433 & 1,001203336 \\
\hline 4 & 1,8760014304 & 1,001258195 \\
\hline 5 & 1,8760366157 & 1,001276975 \\
\hline
\end{tabular}

\section{Conclusões}

O programa computacional desenvolvido convergiu em todos os testes realizados. $\mathrm{O}$ algoritmo de Evolução Diferencial hibridizado com o Método de Superfície de Resposta convergiu para as soluções ótimas com aproximadamente metade do número de gerações que o algoritmo puro. O DEPP determinou a geometria ótima em problemas aplicados à propulsão e aerodinâmica de foguetes, o que demonstra a sua aplicabilidade em problemas práticos de engenharia.

\section{Agradecimentos}

Os autores agradecem o apoio financeiro do CNPq (Conselho Nacional de Desenvolvimento Científico e Tecnológico), AEB (Agência Espacial Brasileira) através do Programa Uniespaço, e CAPES (Coordenação de Aperfeiçoamento de Pessoal de Nível Superior). O terceiro autor é bolsista do CNPq. Pesquisa desenvolvida com auxílio do CENAPAD-UFC.

\section{Referências}

[1] J. D. Anderson, Jr., "Hypersonic and high temperature gas dynamics". McGraw-Hill, New York, 1989.

[2] J. D. Anderson, Jr., "Modern compressible flow: with historical perspective". 3. ed. New York, McGraw-Hill, 1990.

[3] V. Feoktistov, "Differential Evolution: in search of solutions". New York: Springer Science, 2006.

[4] C. R. Maliska, "Transferência de calor e mecânica dos fluidos computacional”, LTC, Rio de Janeiro, 2 ed., 2010.

[5] A. Miele, editor. "Theory of optimum aerodynamic shapes". Academic Press, New York, 1965.

[6] R. Storn; K. Price, Differential evolution: a simple and efficient heuristic for global optimization over continuous spaces. Journal of Global Optimization, Springer Netherlands, v. 11, p. 341-359, 1997.

[7] L. Vincenzi; M. Savoia, Improving the speed performance of an evolutionary algorithm by a second-order cost function approximation. Lisbon: $2^{\text {nd }}$ International Conference on Engineering Optimization, 2010. 